\title{
Risk factors for 30-day hospital readmission after radical gastrectomy: a single-center retrospective study
}

\author{
Raito Asaoka ${ }^{1} \cdot$ Taiichi Kawamura $^{1} \cdot$ Rie Makuuchi $^{1} \cdot$ Tomoyuki Irino $^{1} \cdot$ Yutaka Tanizawa ${ }^{1} \cdot$ Etsuro Bando $^{1}$. \\ Masanori Terashima ${ }^{1}$ (1)
}

Received: 16 May 2018 / Accepted: 10 July 2018 / Published online: 13 July 2018

(c) The International Gastric Cancer Association and The Japanese Gastric Cancer Association 2018

\begin{abstract}
Background Hospital readmission is gathering greater attention as a measure of health care quality. The introduction of fast-track surgery has led to shorter lengths of hospitalization without increasing the risk of postoperative complications and readmission. The collection of comprehensive readmission data is essential for the further improvement of patient care. The aim of the present study is to evaluate the risk factors for readmission within 30 days of discharge after gastrectomy. Methods A total of 1929 patients who underwent radical gastrectomy at Shizuoka Cancer Center were included in this study. A risk analysis with a stepwise logistic regression model was conducted to identify the risk factors for 30-day hospital readmission.

Results The 30-day readmission rate was $2.70 \%$. Common causes of readmission were an intolerance of oral intake and the presence of an intra-abdominal abscess. The $\mathrm{C}$ reactive protein (CRP) level on postoperative day (POD) 3 was significantly higher in the readmitted group; however, the other surgical outcomes, including the incidence of postoperative complications, did not differ to a statistically significant extent. The stepwise logistic regression analysis revealed that CRP on POD3 $\geq 12 \mathrm{mg} / \mathrm{dl}$ [odds ratio (OR) 2.08, 95\% confidence interval (CI) 1.09-3.95, $p=0.025$ ], laparoscopic surgery (OR $2.25,95 \%$ CI 1.17-4.31, $p=0.015$ ), and TG (OR 2.23, 95\% CI 1.17-4.78, $p=0.023$ ) were found to be independent risk factors for readmission.

Conclusions CRP on POD3 $\geq 12 \mathrm{mg} / \mathrm{dl}$, laparoscopic surgery, and TG were identified as independent risk factors for readmission.
\end{abstract}

Keywords Hospital readmission $\cdot$ Radical gastrectomy $\cdot$ Gastric cancer

\section{Introduction}

Fast-track surgery that includes optimized pain control, enforced mobilization and an early oral intake, is currently a main stream of perioperative patient care [1-3], and the length of hospitalization has been reduced. However, as the length of index hospitalization decreases, there are concerns over the need for proper care to avoid complications and

Electronic supplementary material The online version of this article (https://doi.org/10.1007/s10120-018-0856-4) contains supplementary material, which is available to authorized users.

Masanori Terashima

m.terashima@scchr.jp

1 Division of Gastric Surgery, Shizuoka Cancer Center, 1007 Shimonagakubo, Nagaizumi-cho, Sunto-gun, Shizuoka 411-8777, Japan readmission after discharge. Although most studies [4-7] show that early discharge programs are not associated with an increased readmission rate or an increased incidence of postoperative complications in comparison to conventional care, the post-discharge period represents a period of vulnerability in which the patient has a high risk of readmission and complications. In the United States, approximately 15\% of surgical patients in Medicare fee-for-service programs were readmitted within 1 month after the index hospitalization [8]. The readmission rate in patients undergoing colorectal surgery is reported to be $20 \%$ [9], while Acher et al. reported that the readmission rate of patients undergoing gastrectomy was $14.2 \%$ [10].

Readmission significantly increases healthcare costs [11] and can affect the postoperative recovery and quality of life (QOL) of surgical patients. In the United States, Medicare payments for unplanned rehospitalization were estimated to have 
reached approximately $\$ 17.4$ billion in 2004 and accounted for $17 \%$ of the total hospital payments from Medicare. Given the attendant clinical and economic burdens on the healthcare system, the Medicare Hospital Readmission Reduction Program was enacted as a part of the Affordable Care Act of 2010. Since then, much effort has been focused on reducing the rate of surgical readmission. In spite of these efforts, the readmission rate remained constant for 5 years [12], and achievements seem insufficient. Furthermore, among patients who underwent colorectal surgery, readmitted patients were reported to have a worse prognosis than non-readmitted patients [13]. A large retrospective study investigating hospital readmission after gastrectomy in the USA also reported that readmitted patients showed worse survival [14].

These facts underscore the importance of reducing the readmission rate. In addition to the appropriate assessment of patients at the time of discharge, perioperative care, patient education and post-discharge follow-up, a better understanding of the risk factors for readmission will be necessary for achieving this goal; however, previous models for predicting readmission have thus far been unsuccessful in reducing the readmission rate. This might be due to the inclusion of different populations and a wide variety of diseases. Defining the risk factors for readmission in specific diseases will provide a better understanding of the drivers of readmission. There have already been several studies on the incidence of and risk factors for readmission after gastrectomy $[10,14,15]$; however, the data on which these studies are based are very limited. In these studies, the readmission rates ranged from 2.2 to $14.6 \%$, and a wide variety of risk factors for readmission were reported. Since the results of these retrospective studies are divergent, we performed this study with the aim of evaluating the risk factors for readmission within 30 days of discharge after gastrectomy. Additionally, under the hypothesis that a shorter length of index hospitalization would be associated with an increased readmission rate among patients undergoing gastrectomy, we also performed a subgroup analysis to evaluate the incidence of and risk factors for readmission in patients who were discharged within 14 days after gastrectomy. This is because all previous reports included patients who were hospitalized for a long period during the index hospitalization, and the differences between patients with a short hospitalization period and those with relatively longer hospitalization have not been investigated in detail.

\section{Methods}

\section{Patients}

We conducted a retrospective review of the medical records of the patients undergoing elective radical gastrectomy for gastric cancer with curative intent at SCC between January 2010 and December 2016. Patients with gastric remnant cancer, synchronous multiple cancer or patients undergoing robotic surgery were excluded from the study.

A broad panel of clinicopathologic factors that might influence the risk of readmission were examined. The demographic and clinical characteristics that were evaluated included age, sex, preoperative body mass index (BMI), ECOG performance status (PS), pre-existing comorbidities, clinical TNM stage [according to the 7th edition of the American Joint Committee on Cancer Tumor-NodeMetastasis (TNM) classification] and the administration of neoadjuvant chemotherapy. The surgical details, including the procedure (e.g., total gastrectomy), approach (e.g., laparoscopic surgery), extent of lymphadenectomy, concomitant organ resection (e.g., splenectomy), estimated volume of blood loss, postoperative complications (Clavien-Dindo grade $\geq$ II), and the total calorie intake at discharge (routinely assessed by registered dietitians) were also examined.

\section{Clinical pathway}

All patients were routinely managed with the following standardized perioperative clinical pathway. The patients did not receive preoperative mechanical preparation. The choice of procedure was decided according to the tumor location. The extent of lymphadenectomy was decided according the Japanese gastric cancer treatment guidelines 2014 [16]. One or two closed suction drains were placed intraoperatively, and they are extracted on POD 2 or POD3 according to the amylase value of the drained fluid, which was examined on POD1. Patients started taking sips of water on POD2 and a liquid diet on POD3 (the diet gradually progresses from liquid to solid food over 3 days). The decision regarding when to discharge the patient was made based on the results of blood tests performed on POD6. In principle, the patients who were treated according to our clinical pathway were to be discharged between POD8 and POD10; however, this date could be advanced or postponed according to the patient's request.

\section{Outcome measurement}

Readmission was defined as admission as an inpatient within 30 days of discharge from the index hospitalization for radical gastrectomy. The period of 30 days was selected because it has long been considered the standard time interval for similar studies; thus, it allows for the comparison of the outcomes of the present study with those of previous studies. We also investigated the reason for readmission, interval from the discharge to readmission, severity of complications in readmission (distinct from complications during the index hospitalization), administered treatments, and the length of 
the hospital stay on readmission. Although there might have been multiple reasons for readmission, the most significant problem was recorded as the reason for readmission.

We also evaluated a subgroup of patients who were discharged within 14 days after surgery, with the aim of identifying risk factors for readmission in patients who were discharged without serious problems. The reason why we have decided to use POD14 as the cut-off point was that $97.5 \%$ of the patients who did not suffer Grade $\geq$ II postoperative complications were discharged within 14 days after surgery; thus, this seemed to be a reasonable length of hospital stay for patients without severe complications.

\section{Statistical analysis}

Descriptive statistics are expressed as the mean \pm standard deviation (SD) for continuous variables, median \pm range for discrete variables and percentage for categorical variables. Comparisons between the study groups were conducted using Student's $t$ test or Welch's $t$ test for continuous variables, the Mann-Whitney $U$ test for discrete variables, and Fisher's exact test for categorical variables.

A risk analysis with a logistic regression model was conducted to identify the risk factors for 30-day hospital readmission. A backward stepwise elimination procedure was applied, with a criterion of $p \leq 0.10$ to remain in the final model. Potential predictive factors were selected based on previous studies or identified based on the results of the univariate analyses; a chi-squared test was performed to compare dichotomous variables. $P$ values of $<0.05$ were considered to indicate statistical significance. An exploratory analysis was also performed to determine the risk factors for readmission in a subgroup of patients who were discharged within 14 days after surgery. All of the statistical analyses were performed using the $\mathrm{JMP}^{\circledR} 13$ software program (SAS Institute Inc., Cary, NC, USA).

\section{Results}

We retrospectively reviewed the medical records of 2270 patients who underwent elective gastrectomy with curative intent. Patients who were diagnosed with gastric remnant cancer $(n=50)$, synchronous multiple cancer $(n=64)$, or patients undergoing robotic surgery $(n=227)$ were excluded from this study. A total of 1929 patients were enrolled in the final analysis.

The 30-day readmission rate was $2.70 \%(n=52)$. Table 1 shows the type and severity of the complications at readmission. The leading causes of readmission were an intolerance of oral intake and the presence of an intra-abdominal abscess. One patient required intensive care, and four patients underwent a reoperation. The median interval from index hospitalization to readmission was 9 days [interquartile range (IQR) 4-18], and the median length of hospitalization on readmission was 14 days (IQR 10-24). The type and the severity of the complications at readmission, according to the surgical approach, are summarized in Supplemental Table 1.

The patients' demographic and basic characteristics are shown in Table 2. The BMI $\left(\mathrm{kg} / \mathrm{m}^{2}\right)$ values of the readmitted group were significantly higher (Fig. 1). Among the various pre-existing comorbidities, the incidence of cardiovascular disease was significantly higher in the readmitted group. There were no significant differences between the groups with regard to age gender, ECOG PS, clinical TNM stage, or the administration of neoadjuvant chemotherapy.
Table 1 The type and severity of complications at readmission

\begin{tabular}{|c|c|c|c|c|c|c|c|}
\hline & \multicolumn{7}{|c|}{ Severity of complications at readmission } \\
\hline & I & II & IIIa & IIIb & $\mathrm{IVa}$ & $\mathrm{IVb}$ & Total \\
\hline Type of complication & & & & & & & $n=52$ \\
\hline Intolerance of oral intake & 13 & 2 & 0 & 1 & 0 & 0 & 16 \\
\hline Intra-abdominal abscess & 0 & 10 & 4 & 0 & 0 & 1 & 15 \\
\hline Small bowel obstruction & 0 & 4 & 1 & 2 & 0 & 0 & 7 \\
\hline Delayed gastric emptying & 1 & 2 & 0 & 0 & 0 & 0 & 3 \\
\hline Enteritis & 1 & 0 & 1 & 0 & 0 & 0 & 2 \\
\hline Anastomotic ulcer & 1 & 1 & 0 & 0 & 0 & 0 & 2 \\
\hline Anastomotic stricture & 0 & 0 & 2 & 0 & 0 & 0 & 2 \\
\hline Abdominal pain & 0 & 1 & 0 & 0 & 0 & 0 & 1 \\
\hline GERD & 0 & 1 & 0 & 0 & 0 & 0 & 1 \\
\hline Anastomotic leakage & 0 & 0 & 0 & 1 & 0 & 0 & 1 \\
\hline Aspiration pneumonia & 0 & 1 & 0 & 0 & 0 & 0 & 1 \\
\hline Ureterolithiasis & 0 & 1 & 0 & 0 & 0 & 0 & 1 \\
\hline
\end{tabular}

GERD gastroesophageal reflux disease 
Table 2 The patients' demographic and basic characteristics

\begin{tabular}{|c|c|c|c|}
\hline & Readmitted, $n=52$ & Non-readmitted, $n=1877$ & $p$ value \\
\hline Age [median (range)] & $69(31-89)$ & $73(19-91)$ & 0.076 \\
\hline Gender & & & 0.447 \\
\hline Male & $39(75.0 \%)$ & $1316(70.1 \%)$ & \\
\hline Female & $13(25.0 \%)$ & $561(29.9 \%)$ & \\
\hline $\mathrm{BMI}\left(\mathrm{kg} / \mathrm{m}^{2}\right.$, mean $\left.\pm \mathrm{SD}\right)$ & $24.1 \pm 3.79$ & $22.6 \pm 3.28$ & $0.001 *$ \\
\hline \multicolumn{4}{|l|}{ Performance status } \\
\hline 0 & $44(84.6 \%)$ & $1521(81.0 \%)$ & 0.919 \\
\hline 1 & $7(13.5 \%)$ & $310(16.5 \%)$ & \\
\hline 2 & $1(1.9 \%)$ & $42(2.2 \%)$ & \\
\hline 3 & $0(0 \%)$ & $4(0.2 \%)$ & \\
\hline \multicolumn{4}{|l|}{ Type of comorbidity } \\
\hline Hypertension & $27(51.9 \%)$ & $771(41.1 \%)$ & 0.117 \\
\hline Cardiovascular disease & $34(65.4 \%)$ & $872(46.5 \%)$ & $0.007 *$ \\
\hline Respiratory disease & $4(7.7 \%)$ & $68(3.6 \%)$ & 0.127 \\
\hline Liver disease & $2(3.9 \%)$ & $22(1.2 \%)$ & 0.086 \\
\hline Renal disease & $0(0 \%)$ & $12(0.6 \%)$ & 0.560 \\
\hline Diabetes mellitus & $12(23.8 \%)$ & $268(14.3 \%)$ & 0.076 \\
\hline Use of steroids & $1(1.9 \%)$ & $21(1.1 \%)$ & 0.590 \\
\hline Clinical TNM stage & & & 0.486 \\
\hline I & $35(67.3 \%)$ & $1139(60.7 \%)$ & \\
\hline II & $10(19.2 \%)$ & $334(17.8 \%)$ & \\
\hline III & $7(13.5 \%)$ & $365(19.5 \%)$ & \\
\hline IV & $0(0 \%)$ & $39(2.1 \%)$ & \\
\hline $\begin{array}{l}\text { Administration of neoadjuvant } \\
\text { chemotherapy }\end{array}$ & $1(1.9 \%)$ & $77(4.1 \%)$ & 0.431 \\
\hline
\end{tabular}

$B M I$ body mass index, $S D$ standard deviation

*Statistical significance $(p<0.05)$
Fig. 1 A box plot and violin plot of the BMI values

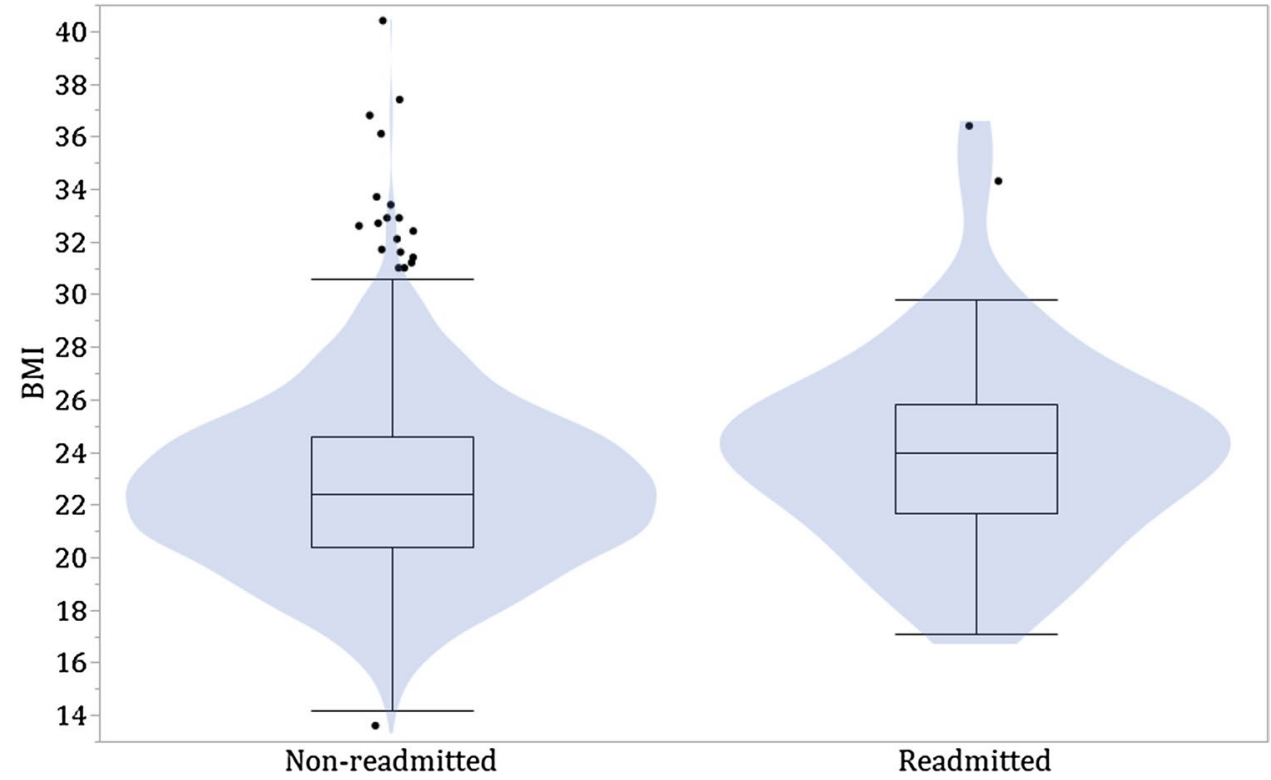


The surgical outcomes are shown in Table 3. The CRP value on POD3 was significantly higher in the readmitted group (Supplemental Fig. 1); however, the other surgical outcomes, including the procedure, approach, extent of lymphadenectomy, and incidence of postoperative complications did not differ to a statistically significant extent. Additionally, when we specifically compared the perioperative CRP values between the two leading causes of readmission (intolerance of oral intake and intra-abdominal abscess) using a repeated measures analysis of variance, the CRP levels of patients who were readmitted due to intra-abdominal abscess were significantly higher $(p=0.039)$. (Supplemental Fig. 2 and Supplemental Table 2).

The results of the univariate analysis are shown in Table 4. The BMI value of $25 \mathrm{~kg} / \mathrm{m}^{2}$ was set as the cut-off point based on the WHO cut-off point for overweight. The cut-off value of CRP on POD3 was determined based on the mean value. The stepwise logistic regression analysis revealed that $\mathrm{CRP}$ on POD $3 \geq 12 \mathrm{mg} / \mathrm{dl}$, laparoscopic surgery, and TG were independent risk factors for readmission (Table 5). The area under the receiver operating characteristic curve (AUC) of this model was 0.705 (sensitivity, 0.59; specificity, 0.77).

The results of the subgroup analysis of patients who were discharged within 14 days after surgery are shown in Supplemental Table 3. A multivariate analysis of the predictors of readmission demonstrated that CRP on POD $3 \geq 12 \mathrm{mg} / \mathrm{dl}$, more than three pre-existing comorbidities, TG and laparoscopic surgery were independent risk factors for readmission. (AUC, 0.710; sensitivity, 0.78; specificity, 0.60).
Table 3 The outcomes of surgery

\begin{tabular}{|c|c|c|c|}
\hline & Readmitted, $n=52$ & Non-readmitted, $n=1877$ & $p$ value \\
\hline Procedure & & & 0.208 \\
\hline Distal gastrectomy & $21(40.4 \%)$ & $1057(56.3 \%)$ & \\
\hline Total gastrectomy & $20(38.5 \%)$ & $499(26.6 \%)$ & \\
\hline Proximal gastrectomy & $4(7.7 \%)$ & $103(5.5 \%)$ & \\
\hline Pylorus preserving gastrectomy & $7(13.5 \%)$ & $209(11.1 \%)$ & \\
\hline Pancreaticoduodenectomy & $0(0 \%)$ & $9(0.5 \%)$ & \\
\hline Approach & & & 0.169 \\
\hline Open surgery & $34(65.4 \%)$ & $1387(73.9 \%)$ & \\
\hline Laparoscopic surgery & $18(34.6 \%)$ & $490(26.1 \%)$ & \\
\hline Extent of lymphadenectomy & & & 0.545 \\
\hline D1 & $0(0 \%)$ & $6(0.3 \%)$ & \\
\hline D1+ & $30(57.7 \%)$ & $921(49.1 \%)$ & \\
\hline D2 & $21(40.4 \%)$ & $930(49.6 \%)$ & \\
\hline $\mathrm{D} 2+$ & $1(1.9 \%)$ & $20(1.1 \%)$ & \\
\hline \multicolumn{4}{|l|}{ Concomitant organ resection } \\
\hline Gall bladder & $8(17.4 \%)$ & $245(13.5 \%)$ & 0.446 \\
\hline Pancreas & $0(0 \%)$ & $44(2.34 \%)$ & 0.264 \\
\hline Spleen & $8(15.4 \%)$ & $269(14.3 \%)$ & 0.832 \\
\hline Liver & $0(0 \%)$ & $18(1.0 \%)$ & 0.478 \\
\hline Colon/small intestine & $1(1.9 \%)$ & $26(1.4 \%)$ & 0.745 \\
\hline Ovary & $0(0 \%)$ & $5(0.3 \%)$ & 0.710 \\
\hline Estimated blood loss [ml, median (range)] & $195(5-1481)$ & $196(5-2708)$ & 0.593 \\
\hline CRP on POD3 (mg/dl, mean \pm SD) & $14.3 \pm 5.95$ & $12.2 \pm 6.08$ & $0.015^{*}$ \\
\hline CRP on POD6 (mg/dl, mean \pm SD) & $7.19 \pm 5.82$ & $5.88 \pm 5.08$ & 0.072 \\
\hline CRP before discharge $(\mathrm{mg} / \mathrm{dl}$, mean $\pm \mathrm{SD})$ & $4.12 \pm 3.31$ & $3.42 \pm 2.57$ & 0.062 \\
\hline Postoperative complication grade $\geq \mathrm{II}^{\mathrm{a}}$ & $17(32.7 \%)$ & $498(26.5 \%)$ & 0.322 \\
\hline Extended use of antibiotics & $11(21.2 \%)$ & $339(18.1 \%)$ & 0.577 \\
\hline Length of postoperative stay (median, IQR) & $10(9,13)$ & $10(8,11)$ & 0.063 \\
\hline Discharge within 14 days after surgery & $42(80.8 \%)$ & $1577(84.0 \%)$ & 0.529 \\
\hline Oral intake $\leq 500 \mathrm{kcal} /$ day at discharge & $7(13.5 \%)$ & $162(8.8 \%)$ & 0.242 \\
\hline
\end{tabular}

$S D$ standard deviation, $C R P C$ reactive protein, $P O D$ postoperative day, $I Q R$ interquartile range

*Statistical significance $(p<0.05)$

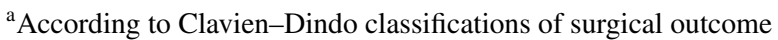


Table 4 The univariate analysis of predictors of readmission

\begin{tabular}{lll}
\hline Variables & OR $(95 \%$ CI $)$ & $p$ value \\
\hline Age $(\geq 75$ vs. $<75)$ & $1.75(0.99-3.08)$ & 0.056 \\
Gender (male vs. female) & $1.28(0.68-2.41)$ & 0.448 \\
BMI ( $\geq 25$ vs. $<25)$ & $2.24(1.27-3.95)$ & $0.006^{*}$ \\
Number of comorbidities ( $\geq 3$ vs. $<3)$ & $2.31(1.28-4.18)$ & $0.006^{*}$ \\
Hypertension & $1.54(0.89-2.69)$ & 0.120 \\
Cardiovascular disease & $2.18(1.22-3.88)$ & $0.008^{*}$ \\
Pulmonary disease & $2.22(0.78-6.32)$ & 0.137 \\
Liver disease & $3.37(0.77-14.7)$ & 0.106 \\
Diabetes mellitus & $1.80(0.93-3.48)$ & 0.080 \\
Procedure (TG vs. others) & $1.73(0.97-3.04)$ & 0.060 \\
Lymph node dissection $(\geq$ D2 vs. $<$ D2) & $0.72(0.41-1.25)$ & 0.242 \\
Approach $($ laparoscopic vs. open) & $1.50(0.84-2.68)$ & 0.172 \\
Oral intake at discharge $<500$ kcal/day vs. $\geq 500 \mathrm{kcal} /$ day & $1.62(0.72-3.64)$ & 0.246 \\
Postoperative complication grade $\geq \mathrm{II}^{\mathrm{a}}$ vs. grade $<\mathrm{II}$ & $1.34(0.75-2.42)$ & 0.324 \\
Discharge within 14 days after surgery & $0.80(0.40-1.61)$ & 0.530 \\
CRP on POD3 $\geq 11$ & $2.54(1.35-4.80)$ & $0.004^{*}$ \\
\hline
\end{tabular}

$O R$ odds ratio, $C I$ confidence interval, $C R P C$ reactive protein; $P O D$ postoperative day

*Statistical significance $(p<0.05)$

${ }^{a}$ According to Clavien-Dindo classification of surgical complications
Table 5 The multivariate analysis of predictors of readmission

\begin{tabular}{lll}
\hline Variables & OR (95\% CI) & $p$ value \\
\hline BMI ( $\geq 25$ vs. $<25)$ & $1.72(0.93-3.20)$ & 0.082 \\
$\begin{array}{l}\text { Total number of comorbidities }(\geq 3 \\
\quad \text { vs }<3)\end{array}$ & $1.79(0.96-3.34)$ & 0.066 \\
Age $(\geq 75$ vs. $<75)$ & $1.72(0.93-3.08)$ & 0.071 \\
Procedure (TG vs. others) & $2.23(1.17-4.23)$ & $0.014^{*}$ \\
Approach (laparoscopic vs. open) & $2.25(1.17-4.31)$ & $0.015^{*}$ \\
CRP on POD3 $\geq 12$ & $2.08(1.09-3.95)$ & $0.025^{*}$ \\
\hline
\end{tabular}

$O R$ odds ratio, $C I$ confidence interval, $C R P \mathrm{C}$ reactive protein, $P O D$ postoperative day

*Statistical significance $(p<0.05)$

\section{Discussion}

In the present study, we identified three independent risk factors for readmission. TG was identified as a risk factor in previous reports $[10,12,17]$. Approximately half of the 20 patients who were readmitted after TG were readmitted due to the intolerance of oral intake. Reduced food intake after TG remains a major problem that is yet to be solved. The impaired oral intake after surgery may be attributed to the lack of a food reservoir $[18,19]$ or decreased ghrelin (a hormone that stimulates appetite [20]) levels. In our facility, registered dieticians routinely provide each patient with nutrition counselling before and after surgery, along with an assessment of the total calorie intake at discharge.
Although insufficient calorie intake $(<500 \mathrm{kcal} / \mathrm{day})$ at discharge was not a risk factor for readmission, it may be noteworthy because three of four patients undergoing TG with an insufficient calorie intake were readmitted due to the intolerance of oral intake. A multi-center prospective study reported that postoperative nutritional support and counselling reduced the rate of readmission [21]. Furthermore, a systematic review and meta-analysis revealed that the administration of oral nutritional supplements during admission and/or after discharge significantly reduced the rate of hospital readmission, particularly in older patient groups [22]. Although these studies have targeted different populations from ours (e.g., patients with any diagnosis, at risk for malnutrition at admission), patients undergoing gastrectomy are also at risk for malnutrition. Thus, the development of a standard protocol for postoperative nutritional management might be valuable.

We also identified a CRP value of $\geq 12 \mathrm{mg} / \mathrm{dl}$ on POD3 as a risk factor for readmission. CRP has been reported to be a reliable systemic inflammatory marker for postoperative complications after gastrectomy [23, 24]. CRP is activated by monocyte-derived IL-6, which is a dominant inflammatory cytokine in the postoperative period. IL-6 not only plays an important role in the acute inflammatory phase, it also suppresses the immune system by several mechanisms. Local immune responses to surgery lead to systemic proinflammatory and immunosuppressive phases that are proportionate in magnitude [25]. The initial inflammatory cytokine spike is presumably high in patients with high CRP levels, and is followed by a proportionately high degree of 
immunosuppression. This mechanism may contribute to the occurrence of complications after discharge.

Another important finding of this study was that laparoscopic surgery was an independent risk factor for readmission. Many clinical studies [26-30] have focused on the comparison of the short-term and long-term outcomes of conventional open surgery and laparoscopic surgery. A retrospective cohort study [30] based on a nationwide registry database in Japan, revealed that the readmission rate of patients undergoing laparoscopic TG for stage I gastric cancer was higher than that of patients undergoing open TG. Late-onset pancreatic fistula may explain this result. In a nationwide cohort study in Japan, the incidence of pancreatic fistula was higher among patients undergoing laparoscopic DG than among those undergoing open DG [31]. During supra-pancreatic lymph node dissection in laparoscopic surgery, the first assistant compresses pancreas dorsally, which can cause the leakage of pancreatic juice. Additionally, cavitation and heat generated from an ultrasonically activated device can injure the pancreatic tissue. This thermal injury may be overlooked because the injury is usually minor and undetectable during the operation; however, it gradually becomes evident several days after surgery.

A large retrospective cohort study showed that nearly $42 \%$ of postoperative complications developed within 30 days after hospital discharge, which more than tripled the risk of reoperation and mortality in surgical patients [32]. Other clinical studies [10,33-35] have reported that the development of complications during the index hospitalization was a risk factor for readmission. Thus, postoperative complications have been indicated as a driver of readmission. On the contrary, the present study and other previous studies have shown that the risk of readmission was not increased in patients who experienced in-hospital complications [17, 36]. Thus, whether the development of postoperative complications is an independent risk factor for readmission remains controversial.

There are conflicting data on the relationship between the length of hospital stay and the risk of readmission following gastrectomy. In a nationwide cohort study that investigated patients undergoing colorectal surgery, an increased length of stay increased the risk of readmission; this was thought to result from postoperative complications [13]. On the contrary, in a retrospective study the examining readmission rate following esophagectomy [37], a decreased length of stay was associated with a higher risk of readmission. In the subgroup of patients who were discharged within 14 days after surgery, the admission rate was $2.59 \%$ and discharge within 14 days was not associated with the increased risk of admission in the overall population. This implies that even if patients suffer postoperative complications, 2 weeks may be sufficient for the postoperative observation and evaluation of the patients, and for the treatment of complications.
The present study is associated with several potential limitations. First, the present study was retrospective in nature. Second, these data were collected from a single high-volume centre with a specialized gastric cancer division. Thus, the readmission rate observed in the present study is unlikely to be generalized. Furthermore, the incidence of, and reasons for readmission may vary between hospitals according to the volume of surgery and surgical practices. Third, the timing of discharge and the decision to readmit the patient was based on a subjective decision by the doctors and the preference of the patient, as no definite criteria for discharge and readmission have been set. Thus, a multicentre prospective cohort study that overcomes these limitations should be conducted in the future.

\section{Conclusion}

This study investigated readmission within 30 days after the index hospital discharge in a large group of patients undergoing gastrectomy. We identified three independent risk factors for readmission. The current risk analysis model showed sufficient accuracy in the prediction of readmission after gastrectomy. Patients with risk factors for readmission require careful perioperative management to prevent readmission.

Acknowledgements This work was supported in part by The National Cancer Center Research and Development Fund (29-A-3).

\section{Compliance with ethical standards}

Conflict of interest The authors declare that they have no conflict of interest.

Ethical standards All procedures followed were in accordance with the ethical standards of the responsible committee on human experimentation (institutional and national) and with the Helsinki Declaration of 1964 and later versions. The ethics committees of SCC (Shizuoka, Japan) reviewed and approved this study (no. 29-J158-29-1-3).

Informed consent Informed consent or a substitute for it was obtained from all patients before being included in the study.

\section{References}

1. Terashima M. The earlier the better? Gastric Cancer. 2014;17(2):197-9.

2. Sugisawa N, Tokunaga M, Makuuchi R, Miki Y, Tanizawa Y, Bando E, et al. A phase II study of an enhanced recovery after surgery protocol in gastric cancer surgery. Gastric Cancer. 2016;19(3):961-7.

3. Makuuchi R, Sugisawa N, Kaji S, Hikage M, Tokunaga M, Tanizawa $\mathrm{Y}$, et al. Enhanced recovery after surgery for gastric cancer and an assessment of preoperative carbohydrate loading. Eur J Surg Oncol. 2017;43(1):210-7. 
4. Abdikarim I, Cao XY, Li SZ, Zhao YQ, Taupyk Y, Wang Q. Enhanced recovery after surgery with laparoscopic radical gastrectomy for stomach carcinomas. World J Gastroenterol. 2015;21(47):13339-44.

5. Adamina M, Kehlet H, Tomlinson GA, Senagore AJ, Delaney CP. Enhanced recovery pathways optimize health outcomes and resource utilization: a meta-analysis of randomized controlled trials in colorectal surgery. Surgery. 2011;149(6):830-40.

6. Mastracci TM, Cohen Z, Senagore A, Members of the Evidence Based Reviews in Surgery G. Canadian Association of General Surgeons and American College of Surgeons Evidence-Based Reviews in Surgery. 24. Fast-track programs in colonic surgery. Systematic review of enhanced recovery programmes in colonic surgery. Can J Surg. 2008;51(1):70-2.

7. Wang D, Kong Y, Zhong B, Zhou X, Zhou Y. Fast-track surgery improves postoperative recovery in patients with gastric cancer: a randomized comparison with conventional postoperative care. J Gastrointest Surg. 2010;14(4):620-7.

8. Jencks SF, Williams MV, Coleman EA. Rehospitalizations among patients in the Medicare fee-for-service program. N Engl J Med. 2009;360(14):1418-28.

9. Basse L, Thorbol JE, Lossl K, Kehlet H. Colonic surgery with accelerated rehabilitation or conventional care. Dis Colon Rectum. 2004;47(3):271-7 (discussion 7-8)

10. Acher AW, Squires MH, Fields RC, Poultsides GA, Schmidt C, Votanopoulos KI, et al. Readmission following gastric cancer resection: risk factors and survival. J Gastrointest Surg. 2016;20(7):1284-94.

11. Selby LV, Gennarelli RL, Schnorr GC, Solomon SB, Schattner MA, Elkin EB, et al. Association of hospital costs with complications following total gastrectomy for gastric adenocarcinoma. JAMA Surg. 2017;152(10):953-8

12. Ahmad R, Schmidt BH, Rattner DW, Mullen JT. Factors influencing readmission after curative gastrectomy for gastric cancer. J Am Coll Surg. 2014;218(6):1215-22.

13. Schneider EB, Hyder O, Brooke BS, Efron J, Cameron JL, Edil BH, et al. Patient readmission and mortality after colorectal surgery for colon cancer: impact of length of stay relative to other clinical factors. J Am Coll Surg. 2012;214(4):390-8 (discussion 8-9).

14. Merchant SJ, Ituarte PH, Choi A, Sun V, Chao J, Lee B, et al. Hospital readmission following surgery for gastric cancer: frequency, timing, etiologies, and survival. J Gastrointest Surg. 2015;19(10):1769-81.

15. Jeong O, Kyu Park Y, Ran Jung M, Yeop Ryu S. Analysis of 30-day postdischarge morbidity and readmission after radical gastrectomy for gastric carcinoma: a single-center study of 2107 patients with prospective data. Medicine (Baltimore). 2015;94(11):e259.

16. Japanese Gastric Cancer A. Japanese gastric cancer treatment guidelines 2014 (ver. 4). Gastric Cancer. 2017;20(1):1-19.

17. Honda M, Hiki N, Nunobe S, Ohashi M, Mine S, Watanabe M, et al. Unplanned admission after gastrectomy as a consequence of fast-track surgery: a comparative risk analysis. Gastric Cancer. 2016;19(3):1002-7.

18. Nomura E, Lee SW, Tokuhara T, Nitta T, Kawai M, Uchiyama K. Functional outcomes according to the size of the gastric remnant and the type of reconstruction following distal gastrectomy for gastric cancer: an investigation including total gastrectomy. Jpn J Clin Oncol. 2013;43(12):1195-202.

19. Nakane Y, Okumura S, Akehira K, Okamura S, Boku T, Okusa T, et al. Jejunal pouch reconstruction after total gastrectomy for cancer. A randomized controlled trial. Ann Surg. 1995;222(1):27-35.

20. Koizumi M, Hosoya Y, Dezaki K, Yada T, Hosoda H, Kangawa $\mathrm{K}$, et al. Serum ghrelin levels partially recover with the recovery of appetite and food intake after total gastrectomy. Surg Today. 2014;44(11):2131-7.

21. Sriram K, Sulo S, VanDerBosch G, Partridge J, Feldstein J, Hegazi RA, et al. A comprehensive nutrition-focused quality improvement program reduces 30 -day readmissions and length of stay in hospitalized patients. JPEN J Parenter Enteral Nutr. 2017;41(3):384-91.

22. Stratton RJ, Hebuterne X, Elia M. A systematic review and metaanalysis of the impact of oral nutritional supplements on hospital readmissions. Ageing Res Rev. 2013;12(4):884-97.

23. Kim EY, Yim HW, Park CH, Song KY. C-reactive protein can be an early predictor of postoperative complications after gastrectomy for gastric cancer. Surg Endosc. 2017;31(1):445-54.

24. Shishido Y, Fujitani K, Yamamoto K, Hirao M, Tsujinaka T, Sekimoto M. C-reactive protein on postoperative day 3 as a predictor of infectious complications following gastric cancer resection. Gastric Cancer. 2016;19(1):293-301.

25. Alazawi W, Pirmadjid N, Lahiri R, Bhattacharya S. Inflammatory and immune responses to surgery and their clinical impact. Ann Surg. 2016;264(1):73-80.

26. Strong VE, Devaud N, Allen PJ, Gonen M, Brennan MF, Coit D. Laparoscopic versus open subtotal gastrectomy for adenocarcinoma: a case-control study. Ann Surg Oncol. 2009;16(6):1507-13.

27. Lu W, Gao J, Yang J, Zhang Y, Lv W, Mu J, et al. Long-term clinical outcomes of laparoscopy-assisted distal gastrectomy versus open distal gastrectomy for early gastric cancer: a comprehensive systematic review and meta-analysis of randomized control trials. Medicine (Baltimore). 2016;95(27):e3986.

28. Kim W, Kim HH, Han SU, Kim MC, Hyung WJ, Ryu SW, et al. Decreased morbidity of laparoscopic distal gastrectomy compared with open distal gastrectomy for stage I gastric cancer: short-term outcomes from a multicenter randomized controlled trial (KLASS01). Ann Surg. 2016;263(1):28-35.

29. Caruso S, Patriti A, Roviello F, De Franco L, Franceschini F, Ceccarelli G, et al. Robot-assisted laparoscopic vs open gastrectomy for gastric cancer: systematic review and meta-analysis. World J Clin Oncol. 2017;8(3):273-84.

30. Kodera Y, Yoshida K, Kumamaru H, Kakeji Y, Hiki N, Etoh T, et al. Introducing laparoscopic total gastrectomy for gastric cancer in general practice: a retrospective cohort study based on a nationwide registry database in Japan. Gastric Cancer. 2018. https://doi. org/10.1007/s10120-018-0795-0.

31. Hiki N, Honda M, Etoh T, Yoshida K, Kodera Y, Kakeji Y, et al. Higher incidence of pancreatic fistula in laparoscopic gastrectomy. Real-world evidence from a nationwide prospective cohort study. Gastric Cancer. 2018;21(1):162-70.

32. Kazaure HS, Roman SA, Sosa JA. Association of postdischarge complications with reoperation and mortality in general surgery. Arch Surg. 2012;147(11):1000-7.

33. Kohlnhofer BM, Tevis SE, Weber SM, Kennedy GD. Multiple complications and short length of stay are associated with postoperative readmissions. Am J Surg. 2014;207(4):449-56.

34. Kelly KN, Iannuzzi JC, Aquina CT, Probst CP, Noyes K, Monson JR, et al. Timing of discharge: a key to understanding the reason for readmission after colorectal surgery. J Gastrointest Surg. 2015;19(3):418-27 (discussion 27-8).

35. Kelly KN, Iannuzzi JC, Rickles AS, Monson JR, Fleming FJ. Risk factors associated with 30-day postoperative readmissions in major gastrointestinal resections. J Gastrointest Surg. 2014;18(1):35-43 (discussion 4).

36. Zhuang CL, Wang SL, Huang DD, Pang WY, Lou N, Chen BC, et al. Risk factors for hospital readmission after radical gastrectomy for gastric cancer: a prospective study. PLoS One. 2015;10(4):e0125572.

37. Sundaram A, Srinivasan A, Baker S, Mittal SK. Readmission and risk factors for readmission following esophagectomy for esophageal cancer. J Gastrointest Surg. 2015;19(4):581-5 (discussion 6). 